\title{
Invariant families of cones and Lyapunov exponents
}

\author{
MACIEJ WOJTKOWSKI \\ Department of Mathematics, University of Arizona, Tucson, AZ 85721, USA
}

(Received 8 November 1983)

Abstract. We show that in several cases preservation of cones leads to non-vanishing of (some) Lyapunov exponents. It gives simple and effective criteria for nonvanishing of the exponents, which is demonstrated on the example of the billiards studied by Bunimovich. It is also shown that geodesic flows on manifolds of non-positive sectional curvature can be treated from this point of view.

\section{Introduction}

Consider a compact manifold $M^{n}$ with a Riemann metric and a diffeomorphism $\phi: M^{n} \rightarrow M^{n}$ preserving probability measure $\mu$. According to the multiplicative ergodic theorem of Oseledec (see [10], [11] and [13]) for almost all $x \in M^{n}$ there is a basis $\left\{e_{1}, \ldots, e_{n}\right\}$ in $T_{x} M^{n}$ and real numbers called Lyapunov characteristic exponents

$$
\chi_{1}(x) \leq \cdots \leq \chi_{n}(x)
$$

such that

$$
\lim _{k \rightarrow \pm \infty} \frac{1}{k} \log \left\|D_{x} \phi^{k}\left(e_{i}\right)\right\|= \pm \chi_{i}(x), \quad i=1, \ldots, n .
$$

If the measure $\mu$ is equivalent to Lebesgue measure then $\mu$ almost everywhere

$$
\chi_{1}(x)+\cdots+\chi_{n}(x)=0
$$

(see [3]).

Presence of non-zero exponents implies positivity of the metric entropy of $\phi$. If all Lyapunov exponents are non-zero almost everywhere then ergodic components of $\phi$ have positive measure and under additional assumptions $\phi$ is Bernoulli (see [11]).

Lyapunov exponents can be put in a more general framework. Consider a probability space $(X, \mu)$ and a measure-preserving transformation $\tau: X \rightarrow X$. Let $A: X \rightarrow \mathrm{GL}(n, \mathbb{R})$ be a measurable mapping to $n \times n$ matrices such that $\log ^{+}\|A(\cdot)\| \in$ $L^{1}(X, \mu)$. Then for $\mu$-almost all $x \in X$ there are subspaces $\{0\}=$ $V_{x}^{0} \subset V_{x}^{\prime} \subset \cdots \subset V_{x}^{n}=\mathbb{R}^{n}$ and numbers $\chi_{1}(x) \leq \cdots \leq \chi_{n}(x)$ such that

$$
\lim _{k \rightarrow+\infty} \frac{1}{k} \log \left\|A\left(\tau^{k-1} x\right) \cdots A(\tau x) A(x) v\right\|=\chi_{i}(x)
$$

if $v \in V_{x}^{i} \backslash V_{x}^{i-1}, i=1, \ldots, n$. 
The classical Alexeev's method ([1]) of establishing non-vanishing of Lyapunov exponents can be described in the following way.

Let $\mathbb{R}^{n}=\mathbb{R}^{r} \times \mathbb{R}^{s}$ and for a given $a>0$ consider a cone

$$
C_{a}=\left\{(u, t) \in \mathbb{R}^{r} \times \mathbb{R}^{s} \mid\|u\| \geq a\|t\|\right\} .
$$

If, for almost all $x \in X$,

(i) $A(x) C_{a} \subset C_{a}$;

(ii) there is $\eta>1$ such that if $v \in C_{a}$ then $\|A(x) v\| \geq \eta\|v\|$; then

$$
0<\log \eta \leq \chi^{n-r+1} \leq \cdots \leq \chi^{n}
$$

This approach, although very simple, is quite efficient in the study of particular dynamical systems. It can be formulated in the setting of a diffeomorphism of a manifold. In this paper we will establish that in several cases the condition (i) alone leads to non-vanishing of (some) Lyapunov exponents. This idea initially appeared in [6].

The plan of the paper is as follows: In $\S 1$ we study non-negative matrices and establish uniform exponential growth of the spectral radius of the product of non-negative matrices from a large family. This section may be of independent interest.

In $\S 2$ we study formal properties of Lyapunov exponents and establish a criterion for positivity of the maximal Lyapunov exponent using the results of $\S 1$.

In $\$ 3$ this criterion is applied to billiards studied by Bunimovich [4] which gives a particularly simple proof that for these billiards Lyapunov exponents are non-zero almost everywhere.

In $\S 4$ and $\S 5$ we show that the non-negative matrices of $\S 1$ can be replaced by other families of matrices preserving some cone. In $\S 5$ we study symplectic matrices and establish a criterion applicable to geodesic flows on manifolds of non-positive sectional curvature.

I wish to thank Prof. Anatole Katok for valuable discussion which led to the appearance of $\S 3$.

\section{Non-negative matrices}

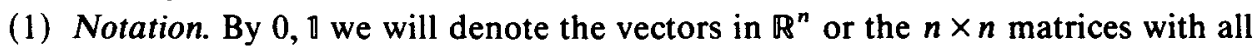
entries equal to 0 and 1 respectively. $I$ denotes the identity matrix. For two $n \times n$ matrices (or vectors in $\mathbb{R}^{n}$ ) $A, B, A \geq B, A>B$ means respectively that every entry of $A$ is $\geq,>$ than the corresponding entry of $B$. If $A \geq B$ we say that $A$ dominates B. Let

$$
0_{n}=\left\{v \in \mathbb{R}^{n} \mid v \geq 0\right\}, \quad 0_{n}^{+}=\left\{v \in \mathbb{R}^{n} \mid v>0\right\}
$$

Further, let

$$
\begin{gathered}
\mathscr{P}=\{A \in \mathrm{GL}(n, \mathbb{R}) \mid A \geq 0\}, \quad \mathscr{P}^{+}=\{A \in \mathrm{GL}(n, \mathbb{R}) \mid A>0\}, \\
S \mathscr{P}=\{A \in \mathscr{P}|| \operatorname{det} A \mid=1\}, \quad S \mathscr{P}^{+}=S \mathscr{P} \cap \mathscr{P}^{+}
\end{gathered}
$$


For $A \in \mathscr{P}, A=\left(a_{i j}\right)_{1 \leq i, j \leq n}$ let $\tilde{A}=\left(\tilde{a}_{i j}\right)_{1 \leq i, j \leq n}$ be a zero-one matrix defined by

$$
\tilde{a}_{i j}= \begin{cases}1 & \text { if } a_{i j}>0 \\ 0 & \text { if } a_{i j}=0 .\end{cases}
$$

Let $\mathscr{P}_{\varepsilon}=\{A \in \mathrm{GL}(n, \mathbb{R}) \| A \geq \varepsilon \mathbb{\|}\}$. Clearly we have $\mathscr{P}^{+}=\bigcup_{\varepsilon>0} \mathscr{P}_{\varepsilon}$. Let $\mathscr{F} \subset \mathscr{P}$ consist of all matrices $A$ such that there are no permutation matrices $P_{1}, P_{2}$ for which $P_{1} A P_{2}$ is a triangular matrix. Obviously $\mathscr{F} \supset \mathscr{P}^{+}$. Finally, $\mathscr{F}_{\varepsilon}=\{A \in \mathscr{F} \mid A \geq \varepsilon \tilde{A}\}$. Hence $\mathscr{F}=\bigcup_{\varepsilon>0} \mathscr{F}_{\varepsilon} . S \mathscr{F}=\{A \in \mathscr{F}|| \operatorname{det} A \mid=1\}$ and $S \mathscr{F}_{\varepsilon}=S \mathscr{F} \cap \mathscr{F}_{\varepsilon}$. By $r(A)$ we denote the spectral norm of $A$. $\|\cdot\|$ denotes the standard euclidean norm in $\mathbb{R}^{n}$ and the corresponding matrix norm. We will assume throughout this section that $n \geq 2$.

(2) Consider the function $F: 0_{n} \rightarrow \mathbb{R}$ defined by $F(v)=v_{1} \cdot \ldots \cdot v_{n}$ where $v=$ $\left(v_{1}, \ldots, v_{n}\right) \cdot(F(v))^{1 / n}$ is a homogeneous function of degree 1 and we will use it to measure the length of a vector in $0_{n}^{+}$.

LEMMA 1.1. If $v \in 0_{n}$ then $\|v\| \geq \sqrt{n}(F(v))^{1 / n}$.

Lemma 1.2. Let $P \in \mathscr{P}$ be a permutation matrix. Then for every $v \in 0_{n}, F(P v)=F(v)$.

Lemma 1.3. Let $D \in \mathscr{P}$ be a diagonal matrix. Then for every $v \in 0_{n}, F(D v)=\operatorname{det} D F(v)$.

For $A \in \mathscr{P}$ we introduce the 'norm' $\rho(A)$ defined by

$$
\rho(A)=\inf _{v \in 0_{n}^{+}}\left(\frac{F(A v)}{F(v)}\right)^{1 / n} .
$$

LeMma 1.4. If $A, B \in \mathscr{P}$ then $\rho(A B) \geq \rho(A) \rho(B)$.

Proof.

$$
\rho(A B)=\inf _{v \in 0_{n}^{+}}\left(\frac{F(A B v)}{F(B v)}\right)^{1 / n}\left(\frac{F(B v)}{F(v)}\right)^{1 / n} \geq \rho(A) \rho(B) .
$$

Lemma 1.5. If $A \in \mathscr{P}$ then $\|A\| \geq \rho(A)$.

Proof. By lemma 1.1,

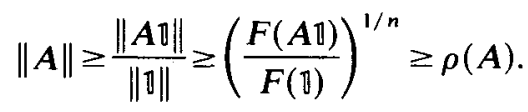

Combining lemmas 1.4 and 1.5 we get

Proposition 1.1. If $A \in \mathscr{P}$ then $r(A) \geq \rho(A)$.

Proof. $\left\|A^{k}\right\| \geq \rho\left(A^{k}\right) \geq(\rho(A))^{k}$. Hence

$$
r(A)=\lim _{k \rightarrow+\infty}\left\|A^{k}\right\|^{1 / k} \geq \rho(A) .
$$

From lemmas 1.2 and 1.3 we get

LeMmA 1.6. If $P_{1}$ and $P_{2}$ are permutation matrices and $A \in \mathscr{P}$, then $\rho\left(P_{1} A P_{2}\right)=\rho(A)$.

LEMMA 1.7. If $A \in \mathscr{P}$ and $D \in \mathscr{P}$ is a diagonal matrix then

$$
\rho(D A)=\rho(A D)=(\operatorname{det} D)^{1 / n} \rho(A) .
$$


We will now formulate a series of propositions giving estimates of $\rho(A)$ for different families of matrices.

Proposition 1.2. If $A \in \mathscr{P}$ then $\rho(A) \geq|\operatorname{det} A|^{1 / n}$.

Proposition 1.3. If $A \in \mathscr{P}_{\varepsilon}$ then $\rho(A) \geq\left(|\operatorname{det} A|+n^{n} \varepsilon^{n}\right)^{1 / n}$.

Proposition 1.4. If $A \in \mathscr{F}_{\varepsilon}$ then $\rho(A) \geq\left(|\operatorname{det} A|+4 \varepsilon^{n}\right)^{1 / n}$.

Proof of propositions 1.2, 1.3 and 1.4. Let $A \in \mathscr{P}$. In view of lemma 1.6, without loss of generality we can assume that $\operatorname{det} A>0$. If $A=\left(a_{i j}\right)_{1 \leq i, j \leq n}$ and $v \in 0_{n}^{+}$,

$$
F(A v)=\left(\sum_{h} a_{1 h(1)} a_{2 h(2)} \cdots a_{n h(n)}\right) F(v)+\text { the other terms }
$$

where the summation is over all permutations $h$ and 'the other terms' are nonnegative. This proves proposition 1.2.

Further, if $A \in \mathscr{F}_{\varepsilon}$,

$$
\begin{aligned}
F(A v) & =\operatorname{det} A F(v)+2\left(\sum_{h \text { odd }} a_{1 h(1)} \cdots a_{n h(n)}\right) F(v)+\text { the other terms } \\
& \geq \operatorname{det} A F(v)+\varepsilon^{n}(F(\tilde{A} v)-\operatorname{det} \tilde{A} F(v))
\end{aligned}
$$

The inequality was obtained by estimating all non-zero entries of $A$ by $\varepsilon$.

For $A \in \mathscr{P}_{\varepsilon}$ we have $\tilde{A}=\nabla$ and det $\tilde{A}=0$. Moreover, by straight-forward calculation, $\rho(\mathbb{\nabla})=n$. Hence $F(\mathbb{v} v) \geq n^{n} F(v)$ and we get proposition 1.3.

To prove proposition 1.4 it is sufficient to prove that for $A \in \mathscr{F}$

$$
\rho(\tilde{A}) \geq(|\operatorname{det} \tilde{A}|+4)^{1 / n}
$$

This will be done in lemmas 1.8 and 1.9 .

LEMMA 1.8. If $Z$ is a zero-one matrix such that $Z$ dominates $I$ (i.e. $Z \geq I$ ) and it does not dominate any other permutation matrix, then there is a permutation matrix $P$ such that $P^{-1} Z P$ is triangular.

Proof. By induction on the dimension of $Z$. For $n=2$ there is nothing to prove. To obtain an inductive step we must prove that under the assumption above there is a column of $Z$ with exactly one 1 (placed obviously on the main diagonal).

The characteristic polynomial of $Z$ is

$$
\operatorname{det}(Z-\lambda I)=(1-\lambda)^{n} \text {. }
$$

Hence the only eigenvalue of $Z$ is equal to 1 . But because $Z \geq 0$, then there must be an eigenvector $v \geq 0, v \neq 0$. We have thus $(Z-I) v=0$. So if, for instance, $v_{1}>0$, then the first column of $Z-I$ is zero.

LeMMA 1.9. If $A \in \mathscr{F}$ then $\rho(\tilde{A}) \geq(|\operatorname{det} \tilde{A}|+4)^{1 / n}$.

Proof. Since det $A \neq 0$ then there is a permutation matrix $P_{1}$ such that $\tilde{A} \geq P_{1}$, i.e.

$$
Z=P_{1}^{-1} \tilde{A} \geq I \text {. }
$$

Since $A \in \mathscr{F}$ then in view of lemma $1.8, Z$ dominates at least one more permutation matrix. By lemma 1.6, $\rho(\tilde{A})=\rho(Z)$. We will get the desired estimate for $\rho(Z)$ by 
induction on the dimension $n$ of $Z, n \geq 2$. For $n=2$,

$$
Z=\left(\begin{array}{ll}
1 & 1 \\
1 & 1
\end{array}\right)
$$

and the estimate is obvious (it is also a special case of proposition 1.3). To obtain the inductive step, consider matrices $Z_{i}, i=1, \ldots, n$ obtained from $Z$ by changing all entries in the first row into zeros except the $i$ th entry which we preserve (some $Z_{i}$ may thus have zero first row). We have, for $v \in 0_{n}$,

$$
F(Z v)=\sum_{i=1}^{n} F\left(Z_{i} v\right)
$$

and

$$
\operatorname{det} Z=\sum_{i=1}^{n} \operatorname{det} Z_{i}
$$

If some $Z_{i_{0}}$ dominates at least two permutation matrices then by inductive assumption and proposition 1.2 ,

$$
\begin{aligned}
F(Z v) & \geq\left(\left|\operatorname{det} Z_{i_{0}}\right|+4\right) F(v)+\sum_{i \neq i_{0}}\left|\operatorname{det} Z_{i}\right| F(v) \\
& \geq(|\operatorname{det} Z|+4) F(v) .
\end{aligned}
$$

Hence we are left with the case when all $Z_{i}$ dominate at most one permutation matrix. Without loss of generality we can assume then that $Z_{1}$ and $Z_{2}$ dominate exactly one permutation matrix and $Z_{1}$ dominates $I$. Then it is clear that $Z_{2}$ can dominate only a cycle. If the length of the cycle is equal to $c \geq 2$ then by a straightforward computation,

$$
F\left(\widetilde{Z_{1}+Z_{2}} v\right) \geq 2^{c} F(v)
$$

and

$$
\operatorname{det}\left(\widetilde{Z_{1}+Z_{2}}\right)= \begin{cases}0 & \text { if } c \text { is even } \\ 2 & \text { if } c \text { is odd }\end{cases}
$$

Hence also in this case,

$$
\begin{aligned}
F(Z v) & =F\left(\widetilde{Z_{1}+Z_{2}} v\right)+\sum_{i=3}^{n} F\left(Z_{i} v\right) \\
& \geq\left(\left|\operatorname{det} \widehat{Z_{1}+Z_{2}}\right|+4\right) F(v)+\sum_{i=3}^{n}\left|\operatorname{det} Z_{i}\right| F(v) \\
& \geq(|\operatorname{det} Z|+4) F(v) .
\end{aligned}
$$

Propositions 1.1 and 1.4 together with lemma 1.4 yield:

Corollary 1.1. If $A_{1}, \ldots, A_{k} \in S \mathscr{F}_{\varepsilon}$ then $r\left(A_{1} \cdots A_{k}\right) \geq\left(1+4 \varepsilon^{n}\right)^{k / n}$.

We are also able to derive from the results above another characterization of the family of matrices $\mathscr{F}$ (and $S \mathscr{F}$ ):

Corollary 1.2. $A \in \mathscr{F}$ if and only if $A \in \mathscr{P}$ and $\rho(A)>|\operatorname{det} A|^{1 / n}$. In particular, $A \in S \mathscr{F}$ if and only if $A \in S \mathscr{P}$ and $\rho(A)>1$. 
Proof. In view of proposition 1.4 and lemmas 1.6 and 1.7, we have only to prove that if $T \in \mathscr{P}$ is a triangular matrix with 1's on the main diagonal, then $\rho(T)=1$.

To this end let $v=(1, \alpha, \ldots, \alpha), \alpha>0$. Then $v \in 0_{n}^{+}$and $\lim _{\alpha \rightarrow 0} F(T v) / F(v)=1$.

From corollary 1.2 and lemma 1.4 we get immediately:

Corollary 1.3. If $A \in \mathscr{P}$ and $B \in \mathscr{F}$ then $A B$ and $B A$ belong to $\mathscr{F}$. In particular, if $A \in S \mathscr{P}$ and $B \in S \mathscr{F}$ then $A B$ and $B A$ belong to $S \mathscr{F}$.

Remark. The privileged role played by the function $F$ in the study of non-negative matrices is no accident. Consider the $n-1$-dimensional projective space $P \mathbb{P}^{n-1}$ and its subset $P 0_{n}$ of lines in $0_{n}^{+}$. Diagonal matrices act freely and transitively in $P 0_{n}^{+}$ and they preserve the measure given by the $(n-1)$-form

$$
\sum_{i=1}^{n}(-1)^{n+1} \frac{v_{i} d v_{1} \wedge \cdots \wedge \widehat{d v}_{i} \wedge \cdots \wedge d v_{n}}{F(v)},
$$

where means that we omit the corresponding 1-form. A non-negative matrix takes $\mathrm{PO}_{n}^{+}$into itself and proposition 1.2 says that it contracts the measure.

\section{Lyapunov exponents}

Let $\tau: X \rightarrow X$ be a measurable transformation preserving a probability measure $\mu$ and let $A: X \rightarrow \operatorname{GL}(n, \mathbb{R})$ be a measurable map such that $\log ^{+}\|A(\cdot)\| \in L^{1}(X, \mu)$. We will call the pair $(\tau, A)$ a measurable cocycle.

For natural $n$, let $A^{n}: X \rightarrow G L(n, \mathbb{R})$ be defined by

$$
A^{n}(x)=A\left(\tau^{n-1} x\right) \cdots A(\tau x) A(x) \text {. }
$$

Thus for any natural $n$ we have a measurable cocycle $\left(\tau^{n}, A^{n}\right)$ which we call the $n$th power of the cocycle $(\tau, A)$. For any $Y \subset X, \mu(Y)>0$, we have the derived transformation $\tau_{Y}: Y \rightarrow Y$. It is defined in the following way: for $x \in Y$, let $k_{Y}(x)=$ $\min \left\{n \geq 1 \mid \tau^{n} x \in Y\right\}$. Then

$$
\tau_{Y} x=\tau^{k_{Y}(x)} x .
$$

We have $k_{Y} \in L^{1}(Y, \mu)$ and the function is called the return time. Let $A_{Y}: Y \rightarrow$ $\mathrm{GL}(n, \mathbb{R})$ be defined by

$$
A_{Y}(x)=A^{k_{Y}(x)}(x) \text {. }
$$

The measurable cocycle $\left(\tau_{Y}, A_{Y}\right)$ will be called the derived cocycle. Given a measurable cocycle $(\tau, A)$ we define for $v \in \mathbb{R}^{n}, v \neq 0$,

$$
\chi(x, v)=\chi(x, v ; \tau, A)=\lim _{l \rightarrow+\infty} \frac{1}{l} \log \left\|A^{l}(x) v\right\| .
$$

By Oseledec's multiplicative ergodic theorem (cf. [10], [13]) the limit above exists almost everywhere, i.e. $\chi(x, v)$ is defined for $\mu$ almost all $x \in X$. It is called the Lyapunov exponent. Moreover, $\chi(x, v)<+\infty$ (the value $-\infty$ is not excluded) and for a given $x \in X, \chi(x, v)$ has at most $n$ distinct values. We have also

$$
\chi_{\max }(x)=\max \left\{\chi(x, v) \mid v \in \mathbb{R}^{n}, v \neq 0\right\}=\lim _{l \rightarrow+\infty} \frac{1}{l} \log \left\|A^{l}(x)\right\| .
$$


LEMMA 2.1. For natural $n, x \in X, v \in \mathbb{B}^{n}, v \neq 0$, we have $\chi\left(x, v ; \tau^{n}, A^{n}\right)=$ $n \chi(x, v ; \tau, A)$.

LeMMA 2.2. Let $Y \subset X, \mu(Y)>0$. Then for $v \in \mathbb{R}^{n}, v \neq 0$ and almost all $x \in Y$,

$$
\chi\left(x, v ; \tau_{Y}, A_{Y}\right)=t(x) \chi(x, v ; \tau, A),
$$

where $t(x)=\lim _{l \rightarrow+\infty}(1 / l) \sum_{i=0}^{l-1} k_{Y}\left(\tau_{Y}^{i} x\right)$, i.e. $t(x)$ is the ergodic mean of the return time $k_{Y}$ with respect to the derived transformation $\tau_{Y}$.

Proof. Let $t_{l}(x)=\sum_{i=0}^{l-1} k_{Y}\left(\tau_{Y}^{i} x\right)$.

$$
\begin{aligned}
\chi\left(x, v ; \tau_{Y}, A_{Y}\right) & =\lim _{l \rightarrow+\infty} \frac{1}{l} \log \left\|\left(A_{Y}\right)^{l} v\right\|=\lim _{l \rightarrow+\infty} \frac{1}{l} \log \left\|A^{t_{l}(x)} v\right\| \\
& =\chi(x, v ; \tau, A) \lim _{l \rightarrow+\infty}\left(t_{l}(x) / l\right) .
\end{aligned}
$$

We will need the following classical lemma from ergodic theory.

LEMMA 2.3. Let $f \in L^{1}(X, \mu)$ be positive almost everywhere. Then $f_{+}=$ $\lim _{l \rightarrow+\infty}(1 / l) \sum_{i=1}^{l-1} f \circ \tau^{i}$ is (defined and) positive almost everywhere.

Proof. We have $f_{+} \geq 0$. Let $Y=\left\{x \in X \mid f_{+}(x)=0\right\}$. Let us assume that $\mu(Y)>0$. Considering $f$ and $\tau$ restricted to $Y$ we get by Birkoff's ergodic theorem, $\int_{Y} f_{+}(x) d \mu=\int_{Y} f(x) d \mu>0$, which gives a contradiction.

THEOREM 2.1. Let $(\tau, A)$ be a measurable cocycle with values in $S \mathscr{F}$, i.e. $A: X \rightarrow S \mathscr{F}$, then the maximal Lyapunov exponent is positive almost everywhere.

Proof. According to corollary 1.2, for all $x, \rho(A(x))>1$ and so $\log \rho(A(x))>0$. Further, by lemmas 1.4 and 1.5 ,

$$
\begin{aligned}
\frac{1}{l} \log \left\|A^{l}(x)\right\| & \geq \frac{1}{l} \log \rho\left(A^{l}(x)\right) \geq \frac{1}{l} \log \rho\left(A\left(\tau^{l-1} x\right)\right) \cdots \rho(A(\tau x)) \rho(A(x)) \\
& =\frac{1}{l} \sum_{i=0}^{l-1} \log \rho\left(A\left(\tau^{i} x\right)\right) .
\end{aligned}
$$

So by lemma 2.3 we get our theorem.

In many cases it is useful to have the following generalization of theorem 2.1.

Theorem 2.2. Let $(\tau, A)$ be a measurable cocycle with values in $S \mathscr{P}$, (i.e. $A: X \rightarrow S \mathscr{P})$ such that for almost every $x \in X$ there is $N(x) \geq 1$ such that $A^{N(x)}(x) \in S \mathscr{F}$. Then the maximal Lyapunov exponent is positive almost everyhere.

Proof. For natural $n$ let $X_{n}=\{x \in X \mid N(x)=n\}$. We have $\bigcup_{n=1}^{+\infty} X_{n}=X$ mod 0. Take $X_{n}$ such that $\mu\left(X_{n}\right)>0$. By corollary 1.3 the measurable cocycle

$$
\left(\left(\tau^{n}\right)_{X_{n}},\left(A^{n}\right)_{X_{n}}\right)
$$

(i.e. the derived cocycle of the $n$th power of $(\tau, A)$ ) has values in $S \mathscr{F}$. Hence by theorem 2.1 its maximal Lyapunov exponent is positive almost everywhere. But then by lemmas 2.1 and 2.2 we get that the maximal Lyapunov exponent of $(\tau, A)$ is positive almost everywhere in $X_{n}$. 
Theorems 2.1 and 2.2 can be put in a setting of a diffeomorphism of a surface (with singularities). Let $\phi: M^{2} \rightarrow M^{2}$ be a diffeomorphism of a two-dimensional manifold $M^{2}$ preserving a probability measure $\mu$ equivalent to an area element $\nu$ defined by some fixed Riemann metric on $M^{2}$ (i.e. $d \mu / d \nu=f$ where $f \in L^{1}\left(M^{2}, \nu\right)$ ). Suppose further that there is a measurable bundle of sectors $C(p) \subset T_{p} M^{2}, p \in M^{2}$ such that

$$
D_{p} \phi(C(p)) \subset C(\phi(p)),
$$

(the sectors $C(p)$ are defined and the invariance property holds only for almost every $p \in M^{2}$ ). Additionally we require that for almost all points $p \in M^{2}$ there is a natural number $n(p)$ such that $D_{p} \phi^{n(p)}(C(p))$ is contained strictly inside $C\left(\phi^{n(p)}(p)\right)$, i.e. the boundary lines of $C(p)$ are mapped inside $C\left(\phi^{n(p)}(p)\right)$. In such a situation we can choose a basis $e_{1}(p), e_{2}(p)$ in almost every tangent plane $T_{p} M^{2}$ so that

$$
C(p)=\left\{v \in T_{p} M^{2} \mid v=a_{1} e_{1}(p)+a_{2} e_{2}(p), a_{1} a_{2} \geq 0\right\},
$$

and the area of the parallelogram spanned by $e_{1}(p), e_{2}(p)$ is equal to $1 / f(p)$. In these coordinates all differentials $D_{p} \phi$ are described by non-negative or non-positive matrices and theorem 2.2 applies except that we must take care of the relation between the norm $\|\cdot\|_{1}$ in $T_{p} M^{2}$ induced from $\mathbb{R}^{2}$ by our choice of basis and the norm $\|\cdot\|$ defined by the Riemann metric. Of course at each point $p \in M$ the two norms are equivalent

$$
\beta(p)\|\cdot\|_{1} \leq\|\cdot\| \leq \alpha(p)\|\cdot\|_{1},
$$

but generally $\alpha, \beta$ are only measurable functions.

By considering $X_{d}=\left\{p \in M^{2} \mid \alpha(p) \leq d, \beta(p) \geq d^{-1}\right\}$, and the derived cocycle we have, in view of lemma 2.2 , that its Lyapunov exponents are zero or non-zero together with those of $(\phi, D \phi)$ and, on the other hand, if we compute them in the norm $\|\cdot\|_{1}$ we get the same result. Hence by theorem 2.2 Lyapunov exponents of $(\phi, D \phi)$ are non-zero almost everywhere.

Having in mind the application to billiards it is important to note that we can allow singularities of $\phi$ without affecting the conclusion (non-vanishing of Lyapunov exponents).

The situation described above appears in several places, among them [8], [12]. We will describe in detail application of these ideas to billiards studied by Bunimovich [4].

\section{Billiards}

We start with some elementary differential geometry. Let $l(t),|t|<\varepsilon$, be a smooth family of directed lines in the plane. We introduce the following infinitesimal characterization of the family along $l(0)$. We define the curvature of the family at a point $p \in l(0)$ to be the curvature with sign at $p$ of the orthogonal section of our family (the orthogonal section is the curve that intersects all the lines of our family orthogonally). We choose the curvature to be negative if the acceleration vector of the section points in the direction of the lines and positive in the other case (see figure 1). If the orthogonal section fails to be a regular curve at $p$ (if it is not defined) then we put $k=\infty$. 


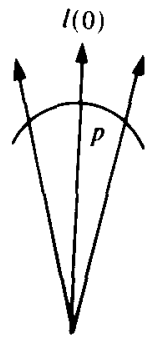

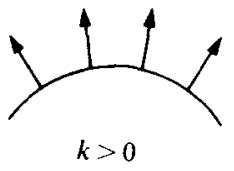

FIGURE 1

Let $k_{1}, k_{2}$ be curvatures of the family at points $p_{1}, p_{2} \in l(0)$. Let $l$ be the distance between $p_{1}$ and $p_{2}$ and the direction of $l(0)$ be from $p_{2}$ to $p_{1}$ (figure 2). Then

$$
k_{2}=\frac{k_{1}}{1-l k_{1}} \text {. }
$$

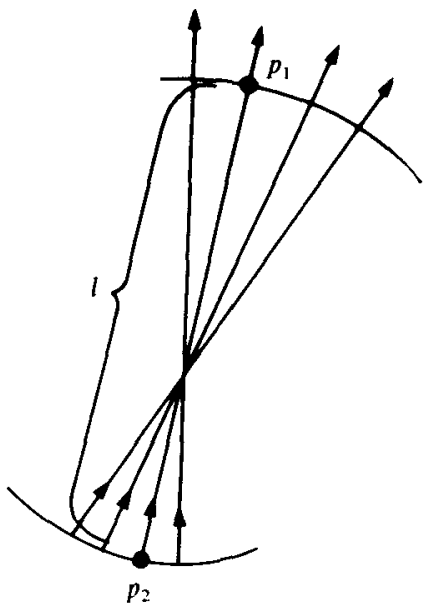

Figure 2

Let us now assume that the family of lines is reflected from some smooth curve. Then at the point of reflection we have two curvatures: $k_{b}$, the curvature of the family before reflection and $k_{\alpha}$, the curvature of the family after reflection. We have

$$
k_{a}=k_{b}+\frac{4}{d}
$$

where $|d|$ is the length of the segment of $l(0)$ inside the curvature disk of the curve at a point of reflection; $d$ is positive if the reflection takes place on the side of the curve on which the curvature disk lies (focusing reflection) and negative in the opposite case (dispersing reflection) (figure 3 ). When the reflection takes place at a point at which the radius of the curvature is infinite (flat point) we have $d=\infty$ and $k_{a}=k_{b}$. 


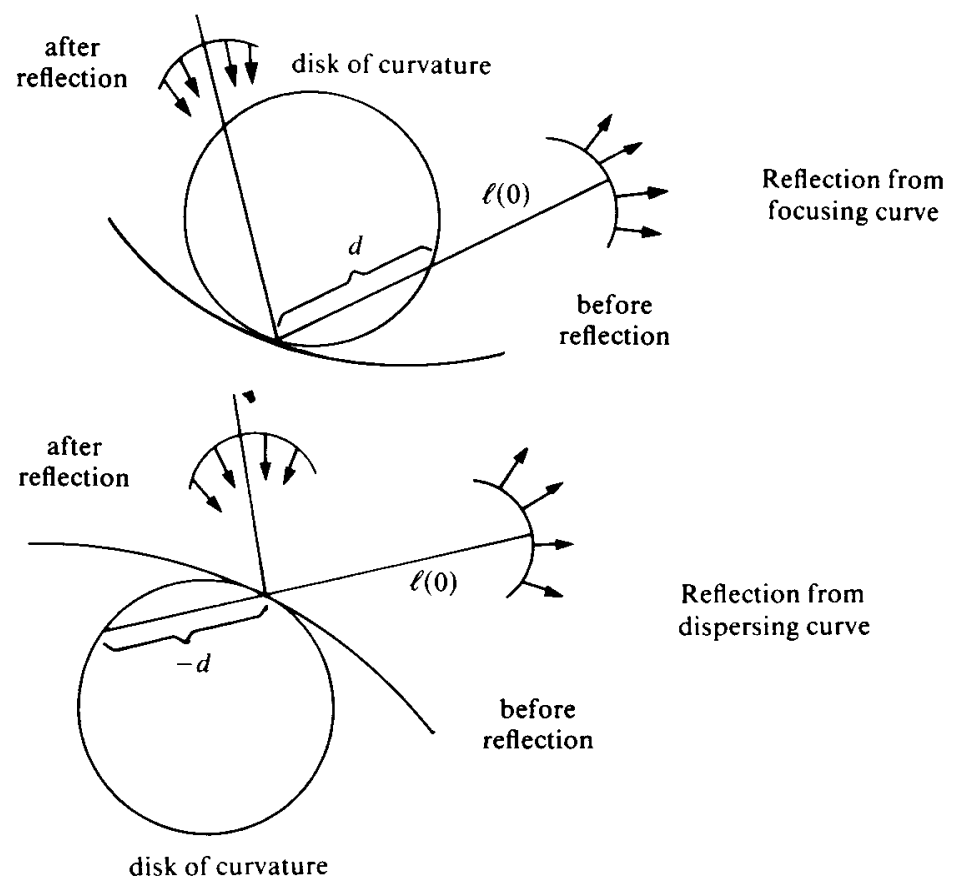

Figure 3

All the above statements can be proved by elementary geometrical considerations and are at least implicit in the literature of the subject (see Bunimovich's paper [4], which contains an extensive bibliography, or [7]).

Consider now a connected domain $Q$ in the plane with piecewise smooth boundary $\partial Q$. We will distinguish between focusing pieces of $\partial Q$-the curvature disk lies on the side of $Q$, and dispersing pieces of $\partial Q$-the curvature disk lies on the other side or is infinite.

By a billiard in $Q$ we mean a dynamical system resulting from the uniform motion of a point mass in $Q$ with reflections at the boundary $\partial Q$ according to the law 'the angle of incidence equals the angle of reflection'. The dynamics of the billiard can be reduced to the transformation $T$ of $S$ where $S$ is the set of unit vectors attached at $\partial Q$ and pointing outward. $T$ is defined in the following way: For $v \in S$ draw a straight line through the point at which $v$ is attached in the direction opposite to that of $v$ up to the next point of intersection with $\partial Q . T v$ is the unit vector attached at this point and symmetric to our straight line with respect to the boundary $\partial Q$ (figure 4). The definition of $T$ follows an established tradition. Clearly $S$ is a two-dimensional manifold with singularities. $T$ preserves a smooth measure (cf. [4]). For almost all points in $S, T$ and all its iterates are differentiable so it makes sense to speak about Lyapunov exponents of $(T, D T)$. A tangent vector to $S$ at $v$ can be viewed as a parametrized family of directed lines $\ell(t),|t|<\varepsilon$, where $\ell(0)$ has the direction of $v$ and passes through the point at which $v$ is attached. Families with the same curvature describe tangent vectors differing by a scalar factor so that 


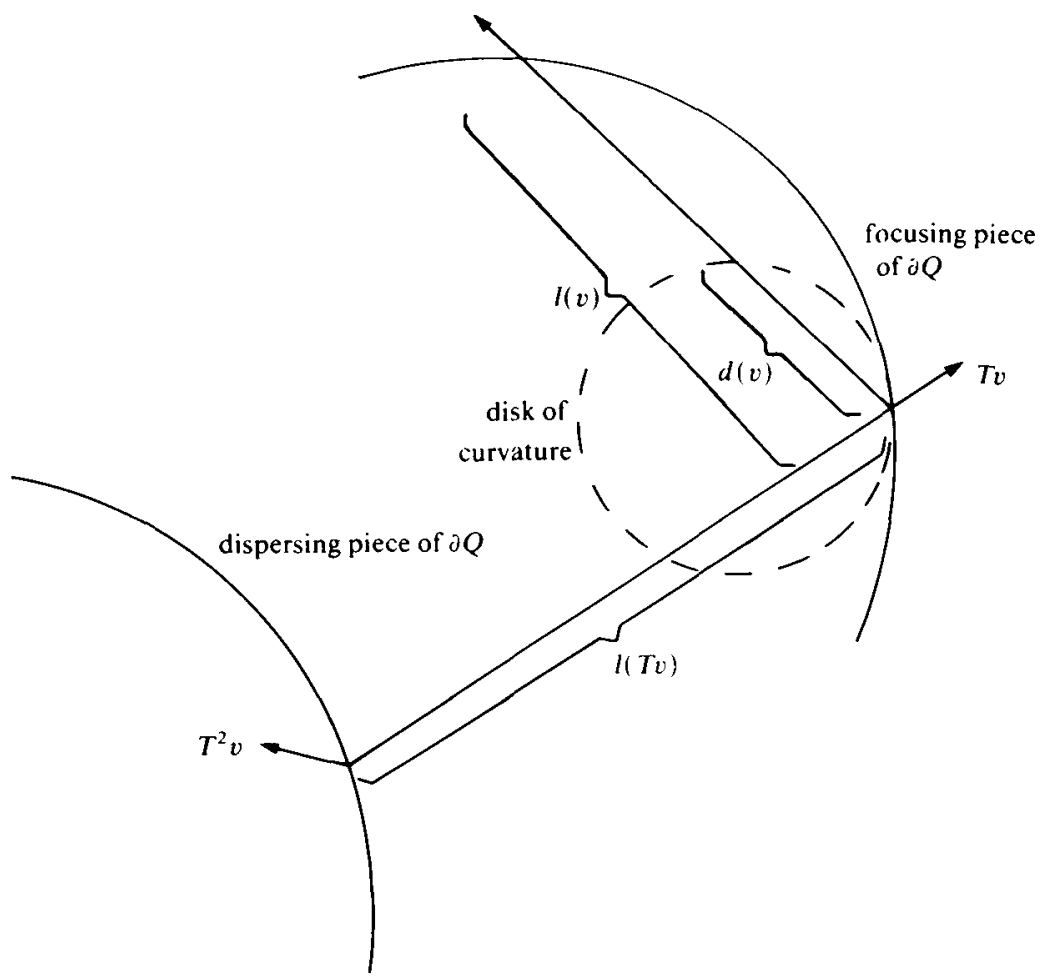

Figure 4

the curvature turns out to be the projective coordinate in the tangent plane of $S$. So, to detect a family of invariant cones for $D T$ we have only to look at the evolution of curvature of a family of lines as it reflects from $\partial Q$ for which we have formulae (1) and (2). Denote projectivization of $D_{v} T$ by $P_{v}$. From (1) and (2) we get

$$
P_{v}(k)=\frac{4}{d(v)}+\frac{k}{1-l(v) k}
$$

where $l(v)$ is the length of the segment of $\ell(0)$ between the point at which $v$ is attached and the next point of intersection with $\partial Q ;|d(v)|$ is the length of the segment of $\ell(0)$ inside the disk of curvature of $\partial Q$ at the latter point; the sign of $d(v)$ is the same as in formula (2) (see figure 4).

We will now assume that $Q$ satisfies Bunimovich conditions:

(a) At every point of a focusing piece of $\partial Q$, the disk of curvature lies entirely in $Q$ (i.e. if $d(v)>0$ then $l(v) \geq d(v)$ in formula (3)).

(b) For almost all $v \in S$ there is $n(v)>0$ such that $d\left(T^{n(v)} v\right)>0$ and $l\left(T^{n(v)} v\right)>$ $d\left(T^{n(v)} v\right)$ or $d\left(T^{n(v)} v\right)<0$.

Remark. The only focusing pieces of $\partial Q$ that are allowed under condition (a) are arcs of circles. Indeed if a curve has variable curvature then except for critical points of the curvature the disk of curvature fails to lie locally on one side of the curve. 
We will now define an invariant bundle of cones. For $v \in S$ we define a cone $C_{v}$ in the tangent plane to $S$ at $v$ by a condition on the curvature $k$ related to a tangent vector:

$$
\begin{array}{ll}
\frac{2}{l(v)} \leq k \leq+\infty & \text { if } v \text { is attached at a focusing piece of } \partial Q \\
-\infty \leq k \leq 0 & \text { if } v \text { is attached at a dispersing piece of } \partial Q .
\end{array}
$$

The bundle of cones $C_{v}$ is invariant under $D T$, i.e. $D T_{v}\left(C_{v}\right) \subset C_{T_{v}}$. Indeed let us check it, for example, in the situation when both $v$ and $T v$ are attached at focusing pieces of $\partial Q$. We get by (3) that if $2 / l(v) \leq k \leq+\infty$ then

$$
\frac{2}{l(T v)} \leq \frac{2}{d(v)} \leq \frac{4}{d(v)}-\frac{2}{l(v)} \leq P_{v}(k) \leq \frac{4}{d(v)}-\frac{1}{l(v)}<+\infty .
$$

Hence by theorem 2.2 and the discussion at the end of $\S 2$ we obtain immediately that the Lyapunov exponents are non-zero almost everywhere. The assumption of strict inclusion in theorem 2.2 is ensured by condition (b).

Pesin theory ([11]) does not apply formally to our billiards because of singularities of $S$ and $T$, but the ideas behind it do apply (see [9]). So the properties of the Bunimovich billiards, such as the positivity of metric entropy, and the Bernoullian property for some power of $T$ on each of the countably many ergodic components, can be obtained formally from non-vanishing of Lyapunov exponents. Ergodicity of such billiards (announced in [4]) lies beyond the scope of our approach.

\section{Other types of cones}

We will now consider matrices preserving other types of cones than the positive octant of $\S 1$. Let $Q: \mathbb{R}^{n} \rightarrow \mathbb{R}$ be a non-degenerate quadratic form of the type $(1, n-1)$. Without loss of generality we can assume that

$$
Q(v)=v_{1}^{2}-v_{2}^{2}-\cdots-v_{n}^{2}=(G v, v)
$$

where

$$
G=\left(\begin{array}{cccc}
1 & & & 0 \\
& -1 & & \\
& & \cdot & \\
0 & & & \cdot-1
\end{array}\right),
$$

and $(\cdot, \cdot)$ denotes the standard scalar product. Linear transformations that preserve $Q$ form the Lorentz group $O(1, n-1)$.

Consider the cone $C=\left\{v \in \mathbb{R}^{n} \mid Q(v) \geq 0\right\}$. As in $\S 1$ we introduce the family of matrices

$$
\begin{aligned}
\mathscr{F} & =\{A \in \mathrm{GL}(n, \mathbb{R}) \mid Q(A v)>0 \text { for } v \in C, v \neq 0\}, \\
S \mathscr{F} & =\{A \in \mathscr{F}|| \operatorname{det} A \mid=1\} .
\end{aligned}
$$

We will measure the length of vectors in $C$ by $\sqrt{Q(v)}$.

LEMMA 4.1. If $v \in C$ then $\|v\| \geq \sqrt{Q(v)}$. 
Again we put for $A \in \mathscr{F}$

$$
\rho(A)=\inf _{v \in \text { int } C} \sqrt{\frac{Q(A v)}{Q(v)}} .
$$

Clearly the analogues of lemmas 1.4 and 1.5 hold so that we can conclude that $r(A) \geq \rho(A)$.

Proposition 4.1. If $A \in S \mathscr{F}$ then $\rho(A)>1$.

Proof. For $A \in S \mathscr{F}$ we have to compare the quadratic forms $A^{*} Q,\left(A^{*} Q\right)(v)=Q(A v)$, and $Q$ on $C$. First we prove that there is a Lorentz transformation $L \in O(1, n-1)$ which diagonalizes $A^{*} Q$, i.e. $L^{*} A^{*} Q$ is diagonal. For this purpose consider the family $P C$ of lines contained in $C, P C \subset P \mathbb{P}^{n-1} . P C$ is diffeomorphic to a closed $n$-1-dimensional disk.

For a linear subspace $V \subset \mathbb{R}^{n}$, let $V^{\perp}$ denote its orthogonal complement with respect to the quadratic form $Q$ and $V^{\Delta}$ the orthogonal complement with respect to $A^{*} Q$.

LEMMA 4.2. If $A \in \mathscr{F}$ then for $l \in P C,\left(l^{\Delta}\right)^{\perp} \in$ int $P C$.

Proof. If $A \in \mathscr{F}$ then $A(P C) \subset$ int $P C$ or equivalently

$$
(A(P C))^{\perp} \subset(\text { int } P C)^{\perp} \text {. }
$$

But $V \in(P C)^{\perp}$ iff $V \cap$ int $C=\varnothing$ so that if $A \in \mathscr{F}$ then

Now for $l \in P C$,

$$
A^{-1}\left((P C)^{\perp}\right) \subset(\text { int } P C)^{\perp} \text {. }
$$

$$
\left(I^{\Delta}\right)^{\perp}=\left(A^{-1}\left((A l)^{\perp}\right)\right)^{\perp} .
$$

So the mapping $P C \ni l \mapsto\left(l^{\Delta}\right)^{\perp} \in$ int $P C$ has a fixed point $l_{0} \in$ int $P C$, i.e. $l_{0}^{\Delta}=l_{0}^{\perp}$. The Lorentz group acts transitively on int $P C$ so that there is a Lorentz transformation $L_{1}$ that maps the $v_{1}$-axis onto $l_{0}$ and hence also the subspace $v_{1}=0$ onto $l_{0}^{\Delta}$. We have as a result

$$
Q\left(A L_{1} v\right)=\lambda_{1} v_{1}^{2}-\left(H v^{\prime}, v^{\prime}\right)
$$

where $H$ is some symmetric positive definite $(n-1) \times(n-1)$ matrix, $v^{\prime}=\left(v_{2}, \ldots, v_{n}\right)$. There is an orthogonal matrix $U$ such that

$$
U^{T} H U=\Lambda^{\prime}=\left(\begin{array}{lll}
\lambda_{2} & & \\
& & \\
& \cdot & \\
0 & & \cdot \lambda_{n}
\end{array}\right)
$$

Clearly $L_{2}$, defined by $L_{2} v=\left(v_{1}, U v^{\prime}\right)$, is a Lorentz transformation. For $L=L_{1} L_{2}$ we get

$$
Q(A L v)=\lambda_{1} v_{1}^{2}-\lambda_{2} v_{2}^{2}-\cdots-\lambda_{n} v_{n}^{2} .
$$

Since $|\operatorname{det} A|=1$ we must have $\lambda_{1} \cdot \ldots \cdot \lambda_{n}=1$. But $Q(A L v)>0$ if $Q(L v)=Q(v) \geq 0$ so that $\lambda_{1}>\max \left\{\lambda_{2}, \cdots, \lambda_{n}\right\}$. Thus $\lambda_{1}>1$ and there is $\eta>1$ such that $\lambda_{1}>\eta>$ $\max \left\{\lambda_{2}, \cdots, \lambda_{n}\right\}$. Finally,

$$
Q(A L v)-\eta Q(L v)=\left(\lambda_{1}-\eta\right) v_{1}^{2}+\left(\eta-\lambda_{2}\right) v_{2}^{2}+\cdots+\left(\eta-\lambda_{n}\right) v_{n}^{2} \geq 0 .
$$

It follows immediately that $\rho(A)>\sqrt{\eta}>1$. 
Remark. Proposition 4.1 is false if we start with a quadratic form $Q$ of type $(k, n-k)$, $2 \leq k \leq n-2$.

Using proposition 4.1 we can conclude in the way described in $\S 2$ that for a measurable cocycle with values in $S \mathscr{F}$ the maximal Lyapunov exponent is positive almost everywhere. For $n=2$ we are back in the framework of $\S 1$ and proposition 4.1 follows from corollary 1.2.

\section{Symplectic Matrices}

Consider the linear symplectic space $\mathbb{R}^{n} \times \mathbb{R}^{n}$ with the standard symplectic form $\omega(u, v)=(\mathscr{J} u, v)$ where

$$
\mathscr{J}=\left(\begin{array}{rr}
0 & -I \\
I & 0
\end{array}\right)
$$

and $(\cdot, \cdot)$ denotes the standard scalar product in $\mathbb{R}^{n} \times \mathbb{R}^{n}$ (or in $\mathbb{R}^{n}$ ).

We take a very special quadratic form $Q$ on $\mathbb{R}^{n} \times \mathbb{R}^{n}$ :

$$
Q(v)=(x, y) \quad \text { where } v=(x, y), \quad x, y \in \mathbb{R}^{n} .
$$

It is a quadratic form of the type $(n, n)$. Let $C=\left\{v \in \mathbb{R}^{2 n} \mid Q(v) \geq 0\right\}$. We will proceed along the same lines as in $\S 1$ and $\S 4$ but restrict our attention to symplectic matrices only. So let

$$
\mathscr{F}=\{S \in \operatorname{Sp}(n, \mathbb{R}) \mid Q(S v)>0 \quad \text { if } v \in C, v \neq 0\}
$$

We have

LEMMA 5.1. If $v \in C$ then $\|v\| \geq \sqrt{2} \sqrt{Q(v)}$.

Again for $S \in \mathscr{F}$ we put $\rho(S)=\inf _{v \in \text { int } C} \sqrt{Q(S v) / Q(v)}$.

LeMMA 5.2. If $S \in \mathscr{F}$ and $v=(x, x), x \in \mathbb{R}^{n}$ then $\|S v\| \geq \rho(S)\|v\|$.

Proof. $\|S v\| \geq \sqrt{2} \sqrt{Q(S v)} \geq \rho(S) \sqrt{2 Q(v)}=\rho(S)\|v\|$.

Proposition 5.1. If $S \in \mathscr{F}$ then $\rho(S)>1$.

Proof. Note that for $A \in \mathrm{GL}(n, \mathbb{R})$,

$$
\left(\begin{array}{cc}
A^{-1} & 0 \\
0 & A^{T}
\end{array}\right)
$$

is a symplectic matrix and it preserves $Q$. Let

$$
S=\left(\begin{array}{ll}
A & B \\
C & D
\end{array}\right)
$$

where $A, B, C, D$ are $n \times n$ matrices. We claim that if $S \in \mathscr{F}$ then $A$ is invertible. Indeed if there is $x \in \mathbb{R}^{n}$ such that $A x=0$ then for $u=(x, 0), Q(u)=0$ and $Q(S u)=0$, thus contradicting the fact that $S$ takes the cone $C$ strictly into itself. So we can consider

$$
S_{1}=\left(\begin{array}{cc}
A^{-1} & 0 \\
0 & A^{T}
\end{array}\right) S
$$


$S_{1}$ is symplectic, i.e. $S_{1}^{T} \mathscr{J} S_{1}=\mathscr{J}$ and we get easily that

$$
S_{1}=\left(\begin{array}{cc}
I & R \\
P & I+P R
\end{array}\right),
$$

where $P$ and $R$ are symmetric. $S_{1}$ clearly belongs to $\mathscr{F}$ so that for $u=(x, 0), x \in \mathbb{R}^{n}$, $Q\left(S_{1} u\right)=(P x, x)>0$ if $x \neq 0$, i.e. $P$ is positive definite. Also $R$ must be positive definite. Indeed if it is not then there is $y \in \mathbb{R}^{n}$ such that $(R y, y) \leq 0, y \neq 0$. But then for $u=(-R y, y) \in C$ we have $S_{1}(u)=(0, z), z \in \mathbb{R}^{n}$, so that $Q\left(S_{1} u\right)=0$ which contradicts the fact that $S_{1} \in \mathscr{F}$.

For $u=(x, y)$ we have

$$
Q\left(S_{1} u\right)=(x, y)+(R y, y)+(P(x+R y), x+R y),
$$

so that

$$
Q(S u)=Q\left(S_{1} u\right) \geq Q(u),
$$

and if $Q(S u)=Q(u)$ then $u=0$. We get that for $u \neq 0, Q(S u)>Q(u)$. Consider the ratio $\sqrt{Q(S u) / Q(u)}$ for $u \in$ int $C,\|u\|=1$. As $u$ approaches the boundary of $C$, $\|u\|=1$, the ratio tends to infinity so that the infimum is attained at a point from int $C$, i.e. $\rho(S)>1$.

As a byproduct of the above proof we get the following.

Proposition 5.2. A symplectic matrix

$$
S=\left(\begin{array}{ll}
A & B \\
C & D
\end{array}\right)
$$

belongs to $\mathscr{F}$ iff $A^{T} C$ and $B A^{T}$ are positive definite $\left(D^{T} B\right.$ and $C D^{T}$ are positive definite).

Note that $A^{T} C$ and $B A^{T}$ (also $D^{T} B$ and $C D^{T}$ ) are symmetric because $S$ is symplectic.

THEOREM 5.1. If a measurable cocycle has values in $\mathscr{F}$ then all its Lyapunov exponents are different from zero.

Proof. By lemma 5.2 and proposition 5.2, using the methods of $\S 2$, we have exponential growth on the whole $n$-dimensional subspace $\left\{(x, y) \in \mathbb{R}^{2 n} \mid x=y\right\}$. So the cocycle has $n$ positive Lyapunov exponents. But if a cocycle has values in symplectic matrices then the Lyapunov exponents appear in pairs $\lambda,-\lambda([3])$. Hence we also have $n$ negative exponents.

There is an infinitesimal version of theorem 5.1. We will formulate it explicitly because it is related to non-vanishing of Lyapunov exponents for geodesic flows on manifolds of non-negative sectional curvature satisfying additional property (rank 1) (see [2] and [5]).

Let us consider a continuous flow $\phi^{\prime}: M \rightarrow M$ on a compact manifold $M$ preserving a probability measure $\mu$ and a continuous mapping $L: M \rightarrow\lrcorner \not(n, \mathbb{R})$, where $\lrcorner h(n, \mathbb{R})$ is the Lie algebra of $\operatorname{Sp}(n, \mathbb{R})$. For $p \in M$ consider the linear differential equation

$$
\frac{d u}{d t}=L\left(\phi^{t}(p)\right) u, \quad u \in \mathbb{R}^{2 n}
$$


General solutions of $(*)$ have the form $u(t)=S^{t} u(0)$ where $S^{t}$ is the matrix solution of $d S / d t=L\left(\phi^{t}(p)\right) S$ such that $S^{0}=I$.

Clearly all matrices $S^{t}, t \in \mathbb{R}$ are symplectic. We can define Lyapunov exponents by

$$
\chi(p, u)=\lim _{t \rightarrow+\infty} \frac{1}{t} \log \left\|S^{t} u\right\| \quad \text { where } p \in M, u \in \mathbb{R}^{2 n} .
$$

By Oseledec's multiplicative ergodic theorem we know that the limit exists $\mu$-almost everywhere and for a given $p \in M, \chi(p, u)$ has at most $2 n$ different values

$$
\lambda_{-n} \leq \cdots \leq \lambda_{-1} \leq \cdots \leq \lambda_{n},
$$

and because $S^{t}$ are symplectic then $\lambda_{-n}=-\lambda_{n}$ (for the last assertion see [3]).

Let us write $L\left(\phi^{t}(p)\right)$ in the form

$$
L\left(\phi^{t}(p)\right)=\left(\begin{array}{cc}
G(t) & N(t) \\
K(t) & -G^{T}(t)
\end{array}\right),
$$

where $K$ and $N$ are symmetric matrices because $L \in\lrcorner \not n(n, \mathbb{R})$. We will assume that both $K$ and $N$ are non-negative definite for all $t \in \mathbb{R}$. This condition is equivalent to $S^{t}\left(S^{s}\right)^{-1} C \subset C$ for all $t \geq s$. Indeed if $u(t)=(x(t), y(t))$ is the solution of $(*)$ then

$$
\begin{aligned}
\frac{d}{d t} Q(u(t)) & =\left(\frac{d x}{d t}, y\right)+\left(x, \frac{d y}{d t}\right)=(G x+N y, y)+\left(x, K x-G^{T} y\right) \\
& =(K x(t), x(t))+(N y(t), y(t)) \geq 0 .
\end{aligned}
$$

Hence if $u \in C$ then $Q(u) \geq 0$ and for $t \geq s$,

$$
Q\left(S^{t}\left(S^{s}\right)^{-1} u\right) \geq Q\left(S^{s}\left(S^{s}\right)^{-1} u\right)=Q(u) \geq 0,
$$

i.e. $S^{t}\left(S^{s}\right)^{-1} C \subset C$. Vice versa if $S^{t}\left(S^{s}\right)^{-1} C \subset C$ for all $t \geq s$, then for $u=(x, y), Q(u)=0$ we have

$$
\left.\frac{d}{d t} Q\left(S^{\prime}\left(S^{s}\right)^{-1} u\right)\right|_{t=s}=(K(s) x, x)+(N(s) y, y) \geq 0
$$

Hence taking $u=(x, 0)$ and $u=(0, y)$ we get that both $K(s)$ and $N(s)$ are non-negative definite. We will say that the pair $\left(\phi^{\prime}, L\right)$ has 'rank 1 ' if additionally it satisfies the following condition:

For almost all $p \in M$ there are no solutions of $(*) u(t)=(x(t), y(t))$ such that $K(t) x(t) \equiv 0$ and $N(t) y(t) \equiv 0$ except for the trivial zero solution.

It is not difficult to see that the last condition can be replaced by:

For almost all $p \in M$ there is $t(p)>0$ such that if $Q(u) \geq 0, u \neq 0$, then $Q\left(S^{\prime(p)} u\right)>$ 0 , i.e. $S^{t(p)}$ takes the cone $C$ strictly into itself.

THEOREM 5.2. If the pair $\left(\phi^{\prime}, L\right)$ as described above has 'rank 1' then all Lyapunov exponents are different from zero.

Proof. The Lyapunov exponents for $\left(\phi^{t}, L\right)$ are the same as for the measurable cocycle $\left(\phi^{1}, S^{\prime}\right)$. Similarly to the proof of theorem 2.2 , let $M_{N}=\{p \in M \mid t(p) \leq N\}$ for natural $N$. We can apply theorem 5.1 to the derived cocycle $\left(\left(\phi^{N}\right)_{M_{N}}\left(S^{N}\right)_{M_{\mathrm{v}}}\right)$ (note that $S^{t} \in \mathscr{F}$ if $t \geq t(p)$ ). But then also for $\left(\phi^{t}, L\right)$ the Lyapunov exponents are non-zero almost everywhere. 
Theorem 5.2 can be used to derive the fact that for geodesic flows on manifolds of non-negative sectional curvature with the rank 1 Riemann metric the Lyapunov exponents are non-zero (except for one corresponding to the direction of the flow) (see [2], [5]). In the case of the geodesic flow,

$$
L=\left(\begin{array}{cc}
0 & I \\
-K & 0
\end{array}\right),
$$

where $-K$ is non-negative definite. The rank 1 condition for the Riemann metric implies that for almost all points there are no solutions of $(*) u(t)=(x(t), 0)$ such that $K(t) x(t) \equiv 0$.

\section{REFERENCES}

[1] V. M. Alekseev. Quasi random dynamical systems. Mat. USSR Sbornik 7 (1969), 1-43.

[2] W. Ballmann \& M. Brin. On the ergodicity of geodesic flows. Ergod. Th. \& Dynam. Sys. 2 (1982), $311-315$.

[3] G. Benettin, I. Galgani, A. Giorgilli \& J.-M. Strelcyn. Lyapunov characteristic exponents for smooth dynamical systems and for Hamiltonian systems; a method for computing all of them. Part 1: theory. Meccanica (1980), 9-20.

[4] L. A. Bunimovich. On the ergodic properties of nowhere dispersing billiards. Commun. Math. Phys. 65 (1979), 295-312.

[5] K. Burns. Hyperbolic behaviour of geodesic flows on manifolds with no focal points. Ergod. Th. \& Dynam. Sys. 3 (1983), 1-12.

[6] E. Cornelis \& M. Wojtkowski. A criterion for the positivity of the Lyapunov characteristic exponent. Preprint (1983).

[7] G. Gallavotti. Lectures on billiards. In Lecture Notes in Physics, 38, Springer (1975).

[8] A. Katok. Bernoulli diffeomorphisms on surfaces. Ann. Math. 110, (1979), 529-547.

[9] A. Katok \& J.-M. Strelcyn. Invariant manifolds for smooth maps with singularities, I, II. Preprint (1981).

[10] V. I. Oseledec. The multiplicative ergodic theorem. The Lyapunov characteristic numbers of dynamical systems. Trans. Mosc. Math. Soc. 19 (1968), 197-231.

[11] Ya. B. Pesin. Lyapunov characteristic exponents and smooth ergodic theory. Russ. Math. Surveys 32 (1977), 55-114.

[12] F. Przytycki. Examples of conservative diffeomorphisms of the two-dimensional torus with coexistence of elliptic and stochastic behaviours. Ergod. Th. \& Dynam. Sys. 2, (1982), 439-463.

[13] D. Ruelle. Ergodic theory of differentiable dynamical systems. Publ. Math. IHES 50 (1979), 27-58. 